\title{
Association of MMP-2, RB and PAI-1 with decreased recurrence- free survival and overall survival in bladder cancer patients
}

\author{
Owen T.M. Chan ${ }^{1, *}$, Hideki Furuya ${ }^{1, *}$, Ian Pagano ${ }^{2}$, Yoshiko Shimizu ${ }^{1,3}$, Kanani \\ Hokutan $^{1,3}$, Lars Dyrskjøt ${ }^{4}$, Jørgen Bjerggaard Jensen ${ }^{5}$, Per-Uno Malmstrom ${ }^{6}$, \\ Ulrika Segersten ${ }^{6}$, Filip Janku ${ }^{7}$ and Charles J. Rosser ${ }^{1}$ \\ ${ }^{1}$ Clinical and Translational Research Program University of Hawaii Cancer Center, Honolulu, HI, USA \\ ${ }^{2}$ Cancer Prevention and Control Program Research Program University of Hawaii Cancer Center, Honolulu, HI, USA \\ ${ }^{3}$ Department of Molecular Biosciences and Bioengineering, University of Hawaii at Manoa, Honolulu, HI, USA \\ ${ }^{4}$ Department of Molecular Medicine, Aarhus University Hospital, Aarhus, Denmark \\ ${ }^{5}$ Department of Urology, Aarhus University Hospital, Aarhus, Denmark \\ ${ }^{6}$ Departments of Surgical Sciences, Uppsala University, Uppsala, Sweden \\ ${ }^{7}$ Department of Investigational Cancer Therapeutics, University of Texas MD Anderson Cancer Center, Houston, TX, USA \\ * Co-first authors
}

Correspondence to: Charles J. Rosser, email: cjrosser@hawaii.edu

Keywords: angiogenin, bladder cancer, IHC, MMP-2, PAI-1, survival

Received: May 07, $2017 \quad$ Accepted: July 12, $2017 \quad$ Published: September 06, 2017

Copyright: Chan et al. This is an open-access article distributed under the terms of the Creative Commons Attribution License 3.0 (CC BY 3.0), which permits unrestricted use, distribution, and reproduction in any medium, provided the original author and source are credited.

\section{ABSTRACT}

Background: We previously reported an accurate urine-based bladder cancer (BCa)-associated diagnostic signature that can be used to non-invasively detect $\mathrm{BCa}$. In this study, we investigated whether a component of this signature could risk stratify patients with $\mathrm{BCa}$.

Methods: Utilizing immunohistochemistry, we investigated angiogenin, MMP2, p53, RB and PAI-1 expression from 939 patients with BCa. The expression levels were scored by assigning a proportion score and an intensity score to yield a total staining score for each protein. The expressions of each protein individually and as an aggregate were then correlated with progression-free survival (PFS), cancer-specific survival (CSS) and overall survival (OS).

Results: Differential expressions of these markers were noted in BCa. With multivariate analysis in non-muscle invasive bladder cancer (NMIBC) age, tumor grade portended a worse PFS, while age, tumor grade, nodal status, MMP2, RB and PAI-1 expression portended a worse OS. As for multivariate analysis in muscle invasive bladder cancer (MIBC), age MMP-2 and RB were associated with a worse PFS, while age, nodal status, MMP-2, RB and PAI-1 were associated with a worse OS. Using Kaplan-Meier survival analysis, we noted a significant reduction in OS as more of the five biomarkers were expressed in a tumor. Thus, overall, high expressions of MMP-2, RB and/or PAI-1 in bladder tumors were markers of poor prognosis.

Conclusion: Individually, MMP-2, RB and PAI-1, as well as in aggregate correlated with poor survival in patients with $\mathrm{BCa}$. Thus, patients whose bladder tumors express these biomarkers may benefit from early radical treatment and/or neoadjuvant or adjuvant therapies. 


\section{INTRODUCTION}

Cancer of the urinary bladder is the fourth most common malignancy in men and the ninth most common malignancy in women in the United States [1]. Urothelial carcinomas constitute approximately $90 \%$ of all bladder cancer (BCa) cases [2]. At presentation, more than $80 \%$ of bladder tumors are non-muscle invasive bladder cancer (NMIBC, i.e., Tis, Ta or T1) and the remaining $20 \%$ of bladder tumors are muscle-invasive bladder cancers (MIBC) or metastatic. NMIBC harbors a 5-year survival rate of approximately 94\% [3, 4]; however, approximately $70 \%$ of patients with these lesions develop tumor recurrence within two years of initial diagnosis. The recurrence phenomenon of NMIBC makes it one of the most prevalent cancers worldwide; in America, it is second only to colorectal cancer. Therefore, NMIBC is a significant burden to healthcare systems $[1,5]$. As for MIBC, 5 -year survival rate is approximately $50 \%$, which drops off to $<20 \%$ in the case of metastatic disease $[6,7]$.

Methods to identify NMIBC and MIBC patients, who are at increased risk of disease recurrence and succumbing to disease, have previously relied on conventional histopathologic evaluation. These histopathologic features have failed to properly risk stratify these patients. Thus, prognostic markers are urgently needed to identify high-risk patients who would benefit from early radical treatment and/or neoadjuvant or adjuvant therapies.

In our previous studies, we have coupled highthroughput, discovery-based technology (i.e., genomics and proteomics) with bioinformatics, in order to derive a $\mathrm{BCa}$-associated diagnostic signature that shows promise for the accurate detection of $\mathrm{BCa}$ in voided urine samples [8-11]. In five independent studies, we have validated our 10 biomarkers, which comprise our $\mathrm{BCa}$-associated diagnostic signature [12-16]. Additionally, we have reported the prognostic significance of two of the 10 biomarkers that comprise the $\mathrm{BCa}$-associated diagnostic signature, angiogenin (ANG) and plasminogen activator inhibitor-1 (PAI-1) [17-20]. Furthermore, in-depth molecular analyses of ANG and PAI-1 have identified other key molecules that possess a significant downstream or upstream interplay with them (i.e., MMP-2 and p53 with ANG and RB and p53 with PAI-1) [17-19, 21].

In the present study, we set out to validate the tissue expression of ANG, MMP-2, p53, PAI-1 and RB in human $\mathrm{BCa}$ with immunohistochemistry conducted on tissue microarrays (TMA). Demographic, clinical, disease and treatment characteristics are presented in Table 1. The aims of the validation were to a) analyze the tissue immunoreactivity of these $\mathrm{BCa}$ candidate biomarkers and b) investigate if the expression of these $\mathrm{BCa}$ candidate biomarkers possesses prognostic value in determining recurrence-free survival and overall survival in $\mathrm{BCa}$ patients.

\section{RESULTS}

\section{P53, RB and PAI-1 are preferentially associated with aggressive bladder cancer}

Figure 1 shows representative immunohistochemical stainings for each of the five targets in a high-grade non-muscle invasive tumor. The relationship between immunophenotype for each target and tumor grade is summarized in Table 2. We found the highest expression levels of p53, RB and PAI-1 (total staining score $\geq 3$ ) associated with high-grade disease compared to lowgrade disease $(58 \%$ vs. $27 \%, 85 \%$ vs. $65 \%, 65 \%$ vs. $49 \%$, respectively). Furthermore, the highest expression of MMP-2 was noted with low-grade disease compared to high-grade disease (64\% vs. 52\%). These differences were statistically significant. The relationships between immunophenotype for each target and tumor stage are summarized in Table 3. We found the highest expression levels of p53 and RB in T2 stage tumors (compared to $\mathrm{Ta} /$ Tis stage tumors $(62 \%$ vs. $29 \%$ and $87 \%$ vs. $68 \%$, respectively). Similar to its expression in low-grade tumors, MMP-2 expression was higher in $\mathrm{Ta} / \mathrm{Tis}$ stage tumors compared to $\mathrm{T} 2$ stage tumors $(62 \%$ vs. $47 \%)$. These differences were statistically significant. However, we observed no difference in p53, RB and MMP-2 expression between $\mathrm{T} 2$ and $\mathrm{T} 3 / \mathrm{T} 4$ stage tumors.

\section{MMP-2, p53, RB, PAI-1 are associated with a reduction in recurrence-free survival and overall survival in bladder cancer}

In NMIBC, univariate analysis indicated that age, tumor grade, tumor stage (Ta/Tis vs. T1), expression of p53 and RB predicted progression-free survival. With multivariate analysis, $>65$ years of age (HR $1.67,95 \% \mathrm{CI}$ $1.16-2.40, p=0.006$ ) and high-grade tumor (HR 1.74, 95\% CI 1.04-2.92, $p=0.03$ ) independently predicted a worse progression-free survival (Table 4a). In MIBC, univariate analysis found that age, gender, expression of MMP-2, RB and PAI-1 predicted progression-free survival. With multivariate analysis, $>65$ years of age (HR $1.26,95 \% \mathrm{CI}$ $1.00-1.58, p=0.05$ ), high MMP-2 expression (HR 2.03, $95 \%$ CI $1.28-3.22, p=0.003$ ) and high RB expression (HR 2.31, 95\%CI 1.26-4.23, $p=0.007$ ) independently predicted a worse progression-free survival (Table 4b). Using the Kaplan-Meier survival analysis with the logrank test, we found significantly reduced progression-free survival in NMIBC but not in MIBC when more of the five biomarkers were expressed in a tumor $(p=0.01$ and $p$ $=0.16$, respectively), Figure $2 \mathrm{a}$ and $2 \mathrm{~b}$. As noted in Table 4, three of the five biomarkers (MMP-2, RB and PAI-1) were critical biomarkers influencing survival analysis of NMIBC and MIBC. We noted no difference in cancerspecific survival in NMIBC or MIBC associated with the expression of these five biomarkers (Supplementary 
Table 1: Demographic, clinical, and pathologic characteristics of the 939 subjects comprising the study cohort

\begin{tabular}{|c|c|c|c|}
\hline Features & $\begin{array}{c}\text { Denmark } \\
\mathbf{N}=\mathbf{5 8 7}\end{array}$ & $\begin{array}{l}\text { Sweden } \\
\mathbf{N}=\mathbf{3 5 2}\end{array}$ & $\begin{array}{c}\text { All Patients } \\
\qquad \mathbf{N}=939\end{array}$ \\
\hline \multicolumn{4}{|l|}{ Age (years) } \\
\hline$\leq 65$ & $274(47 \%)$ & $90(26 \%)$ & $364(39 \%)$ \\
\hline$>65$ & $313(53 \%)$ & $256(73 \%)$ & $569(61 \%)$ \\
\hline Unavailable & $0(0 \%)$ & $6(2 \%)$ & $6(1 \%)$ \\
\hline \multicolumn{4}{|l|}{ Gender } \\
\hline Female & $144(25 \%)$ & $85(24 \%)$ & $229(24 \%)$ \\
\hline Male & $443(75 \%)$ & $264(75 \%)$ & $707(75 \%)$ \\
\hline Unavailable & $0(0 \%)$ & $3(1 \%)$ & $3(0 \%)$ \\
\hline \multicolumn{4}{|l|}{ Tumor Grade } \\
\hline Low & $139(24 \%)$ & $84(24 \%)$ & $223(24 \%)$ \\
\hline High & $437(74 \%)$ & $265(75 \%)$ & $702(75 \%)$ \\
\hline Unavailable & $11(2 \%)$ & $3(1 \%)$ & $14(1 \%)$ \\
\hline \multicolumn{4}{|l|}{ Tumor Stage } \\
\hline Ta or Tis & $139(24 \%)$ & $119(34 \%)$ & $258(27 \%)$ \\
\hline $\mathrm{T} 1$ & $133(23 \%)$ & $117(33 \%)$ & $250(27 \%)$ \\
\hline $\mathrm{T} 2$ & $294(50 \%)$ & $89(25 \%)$ & $383(41 \%)$ \\
\hline $\mathrm{T} 3$ or $\mathrm{T} 4$ & $21(4 \%)$ & $23(7 \%)$ & $44(5 \%)$ \\
\hline Unavailable & $0(0 \%)$ & $4(1 \%)$ & $4(0 \%)$ \\
\hline \multicolumn{4}{|l|}{ Lymph Nodes } \\
\hline $\mathrm{N} 0$ or $\mathrm{Nx}$ & $470(80 \%)$ & $341(97 \%)$ & $811(86 \%)$ \\
\hline N1 & $117(20 \%)$ & $11(3 \%)$ & $128(14 \%)$ \\
\hline \multicolumn{4}{|l|}{ Progression } \\
\hline No & $318(54 \%)$ & $230(65 \%)$ & $548(58 \%)$ \\
\hline Yes & $269(46 \%)$ & $110(31 \%)$ & $379(40 \%)$ \\
\hline Unavailable & $0(0 \%)$ & $12(3 \%)$ & $12(1 \%)$ \\
\hline \multicolumn{4}{|c|}{ Follow-up (years) } \\
\hline Median & 6.83 & 3.75 & 5.37 \\
\hline
\end{tabular}

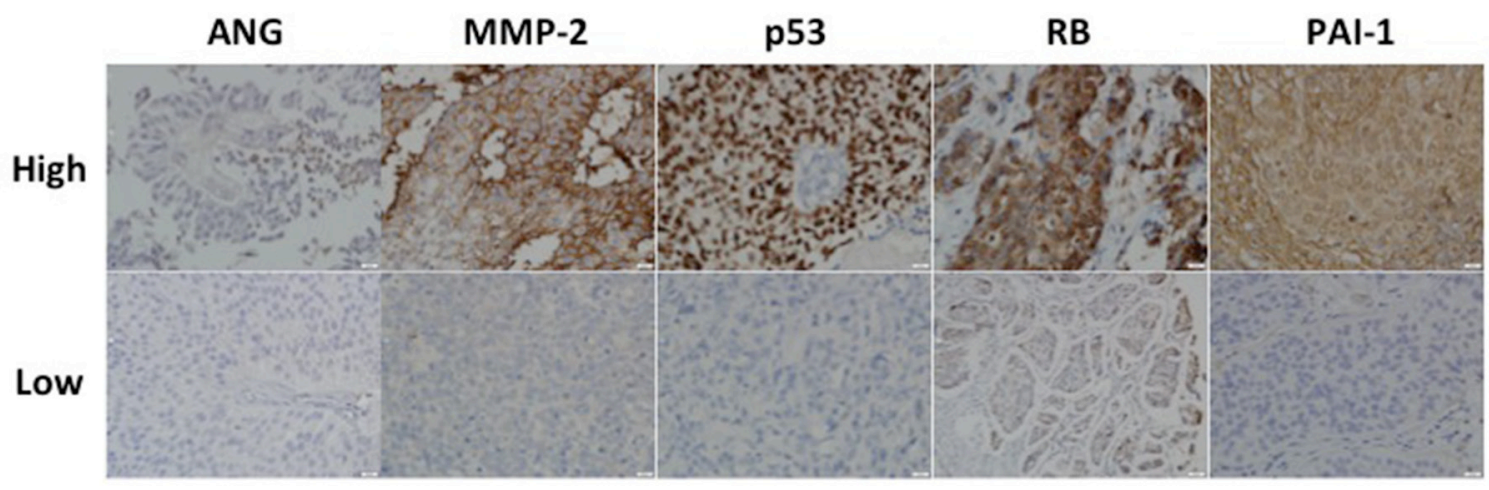

Figure 1: Representative expression status for ANG, MMP-2, p53, RB and PAI-1 in a high-grade non-muscle invasive bladder cancer. Upper row represents high intensity while lower row represents low intensity associated with each target. All images were captured at $400 \times$ magnification. 
Table 2: Relationship between immunochemical features and tumor grade

\begin{tabular}{|c|c|c|c|}
\hline Target Expression & Low-Grade N (Column \%) & High-Grade N (Column \%) & $p$-value* \\
\hline \multicolumn{4}{|l|}{ ANG } \\
\hline $0-2$ & $181(93 \%)$ & $547(92 \%)$ & .64 \\
\hline $3-4$ & $13(7 \%)$ & $48(8 \%)$ & \\
\hline $5-6$ & $0(0 \%)$ & $0(0 \%)$ & \\
\hline \multicolumn{4}{|l|}{ MMP-2 } \\
\hline $0-2$ & $75(36 \%)$ & $286(48 \%)$ & .01 \\
\hline $3-4$ & $129(63 \%)$ & $306(51 \%)$ & \\
\hline $5-6$ & $2(1 \%)$ & $6(1 \%)$ & \\
\hline \multicolumn{4}{|l|}{ p53 } \\
\hline $0-2$ & $158(73 \%)$ & $270(42 \%)$ & $<.0001$ \\
\hline $3-4$ & $55(25 \%)$ & $301(46 \%)$ & \\
\hline $5-6$ & $5(2 \%)$ & $78(12 \%)$ & \\
\hline \multicolumn{4}{|l|}{$\mathrm{Rb}$} \\
\hline $0-2$ & $46(23 \%)$ & $89(15 \%)$ & .008 \\
\hline $3-4$ & $152(76 \%)$ & $508(83 \%)$ & \\
\hline $5-6$ & $1(1 \%)$ & $13(2 \%)$ & \\
\hline \multicolumn{4}{|l|}{ PAI-1 } \\
\hline $0-2$ & $108(51 \%)$ & $228(35 \%)$ & $<.0001$ \\
\hline $3-4$ & $103(48 \%)$ & $400(62 \%)$ & \\
\hline $5-6$ & $2(1 \%)$ & $22(3 \%)$ & \\
\hline
\end{tabular}

*Fisher's Exact Test.

Figure $1 \mathrm{a}$ and $1 \mathrm{~b}$ and Supplementary Table $1 \mathrm{a}$ and $1 \mathrm{~b})$. Lastly, in NMIBC, univariate analysis demonstrated that age, gender, tumor grade, tumor stage (Ta/Tis vs. T1) and expression of MMP-2, RB and PAI-1 predicted overall survival (OS). With multivariate analysis, $>65$ years of age (HR 2.41, 95\% CI 1.78-3.26, $p<0.0001$ ), highgrade tumor (HR 2.11, 95\% CI 1.43-3.12, $p=0.0002$ ) and high expression of MMP-2 (HR 1.87, 95\% CI 1.08-3.26, $p=0.03$ ), RB (HR 3.08, 95\% CI 1.79-5.32, $p=0.0001)$ and PAI-1 (HR 2.58, 95\% CI 1.52-4.38, $p=0.0004$ ) independently predicted a worse OS (Table 5a). Similarly, in $\mathrm{MIBC}$, univariate analysis revealed that age, gender, lymph nodes stage (N0/NX vs. N1) and expression of MMP-2, RB and PAI-1 predicted overall survival (OS). With multivariate analysis, $>65$ years of age (HR 1.38 , 95\% CI 1.10-1.74, $p=0.006)$, lymph nodes N1 stage (HR $1.40,95 \%$ CI 1.0.9-1.80, $p=0.009)$ and high expression of MMP-2 (HR 2.08, 95\% CI 1.29-3.33, $p=0.03$ ), RB (HR $2.18,95 \%$ CI 1.15-4.13, $p=0.02$ ) and PAI-1 (HR 1.69, $95 \%$ CI $1.09-2.62, p=0.02)$ independently predicted a worse OS (Table 5b), while male gender (HR 0.68, 95\% CI $0.54-0.86, p=0.002$ ) independently predicted better
OS. Using the Kaplan-Meier survival analysis with the log-rank test, we noted a significant reduction in OS in both NMIBC and MIBC as more of the five biomarkers were expressed in a tumor $(p=0.001$ and $p=0.03$, respectively), Figure $3 \mathrm{a}$ and $3 \mathrm{~b}$. Thus, the overall, high expression of MMP-2, RB and/or PAI-1 in bladder tumors was a marker of poor prognosis.

\section{DISCUSSION}

In this retrospective analysis, we demonstrated that the expression of MMP-2, RB and PAI-1 correlated with a reduction in recurrence-free survival and overall survival in patients with $\mathrm{BCa}$. Our results also demonstrated the prognostic value of combining ANG, MMP-2, p53, RB and PAI-1 expression for all patients with $\mathrm{BCa}$. Thus, patients whose bladder tumors express these biomarkers may benefit from early radical treatment and/or neoadjuvant or adjuvant therapies. To the best of our knowledge, this represents the largest and most comprehensive study to date demonstrating the prognostic significance of MMP-2, $\mathrm{RB}$ and PAI-1 in patients with $\mathrm{BCa}$. 
Table 3: Relationship between immunochemical features and tumor stage

\begin{tabular}{|c|c|c|c|c|c|}
\hline Target Expression & $\begin{array}{c}\text { Ta or Tis N } \\
\text { (Column \%) }\end{array}$ & $\begin{array}{c}\text { T1 N } \\
(\text { Column \%) }\end{array}$ & $\begin{array}{c}\mathrm{T} 2 \mathrm{~N} \\
(\text { Column \%) }\end{array}$ & $\begin{array}{c}\text { T3 or T4 N } \\
\text { (Column \%) }\end{array}$ & $p$-value ${ }^{*}$ \\
\hline \multicolumn{6}{|l|}{ ANG } \\
\hline $0-2$ & $212(93 \%)$ & $204(94 \%)$ & $283(90 \%)$ & $35(92 \%)$ & .28 \\
\hline $3-4$ & $17(7 \%)$ & $12(6 \%)$ & $32(10 \%)$ & $3(8 \%)$ & \\
\hline $5-6$ & $0(0 \%)$ & $0(0 \%)$ & $0(0 \%)$ & $0(0 \%)$ & \\
\hline \multicolumn{6}{|l|}{ MMP-2 } \\
\hline $0-2$ & $91(38 \%)$ & $86(38 \%)$ & $170(55 \%)$ & $21(55 \%)$ & $<.0001$ \\
\hline $3-4$ & $147(61 \%)$ & $140(62 \%)$ & $132(43 \%)$ & $17(45 \%)$ & \\
\hline $5-6$ & $2(1 \%)$ & $0(0 \%)$ & $6(2 \%)$ & $0(0 \%)$ & \\
\hline \multicolumn{6}{|l|}{ p53 } \\
\hline $0-2$ & $178(71 \%)$ & $112(47 \%)$ & $129(38 \%)$ & $11(26 \%)$ & $<.0001$ \\
\hline $3-4$ & $68(27 \%)$ & $107(45 \%)$ & $160(47 \%)$ & $25(58 \%)$ & \\
\hline $5-6$ & $6(2 \%)$ & $18(8 \%)$ & $53(15 \%)$ & $7(16 \%)$ & \\
\hline \multicolumn{6}{|l|}{$\mathrm{Rb}$} \\
\hline $0-2$ & $57(24 \%)$ & $40(18 \%)$ & $36(11 \%)$ & $3(8 \%)$ & .0001 \\
\hline $3-4$ & $174(75 \%)$ & $181(82 \%)$ & $276(86 \%)$ & $35(88 \%)$ & \\
\hline $5-6$ & $2(1 \%)$ & $1(0 \%)$ & $9(3 \%)$ & $2(5 \%)$ & \\
\hline \multicolumn{6}{|l|}{ PAI-1 } \\
\hline $0-2$ & $109(44 \%)$ & $85(36 \%)$ & $133(38 \%)$ & $9(23 \%)$ & .04 \\
\hline $3-4$ & $136(55 \%)$ & $147(62 \%)$ & $199(57 \%)$ & $30(75 \%)$ & \\
\hline $5-6$ & $4(2 \%)$ & $4(2 \%)$ & $15(4 \%)$ & $1(3 \%)$ & \\
\hline
\end{tabular}

*Fisher's Exact Test.

The biomarkers that comprised this prognostic signature (i.e., ANG, MMP-2, p53, RB and PAI-1) have a varied range of ascribed functions including angiogenesis, breakdown of extracellular matrix, serine protein inhibitor, DNA binding and transcription factor. ANG and PAI-1 [22-26] have also been associated with angiogenesis. Angiogenesis, the development of new blood vessels from existing blood vessels, is essential for normal growth and the development of tissues and organs. Furthermore, in addition to the degradation extracellular matrix by MMP-2, recent studies have suggested that ANG and PAI-1 can breakdown the extracellular matrix $[27,28]$. Degradation of the extracellular matrix allows cells to become more motile. Thus, these extracellular matrix-degrading proteins may work in conjunction with an increase in vasculature, thereby, increasing the probability that motile-invasive tumor cells may enter the circulation to disseminate to distant organs [29]. P53 has both transactivation and transrepression activity and, thus, controls the transcription of numerous genes [30-32]. RB also controls the expression of numerous genes although it does so primarily by recruiting transcription factors and chromatin remodelling proteins [33-35]. Furthermore, we have identified that ANG regulates MMP-2 [17], as well as PAI-1 and p53 expression, [36] while PAI-1 activity is controlled by $\mathrm{p} 53$ and RB [unpublished data].

Previous in vitro and in vivo analyses of ANG and PAI1 began to unravel their potential contributions to $\mathrm{BCa}$ pathobiology. Briefly, there is clear evidence that ANG expression positively regulates MMP-2 expression via ERK1/2 signaling [17]. In addition, ANG regulates MMP-2 expression through two major pathways, a) p53 regulation via ERK pathway activation and $\mathrm{b}$ ) induction of hypomethylation by DNMT3b down-regulation through the ERK pathway [36]. MMPs are known to affect extracellular matrix (ECM) remodeling, angiogenesis, apoptosis, epithelial-to-mesenchymal transition and cell proliferation and, thus, induce aggressive invasion and metastasis of different cancer types, including $\mathrm{BCa}$ [37]. Assessing the GEO database, we found that MIBC specimens had increased levels of ANG and MMP-2 compared to NMIBC. These findings suggest ANG may 
Table 4a: Progression Free Results for Non-Muscle Invasive Bladder Cancer

\begin{tabular}{|c|c|c|c|c|c|c|c|c|c|c|c|c|}
\hline & \multicolumn{6}{|c|}{ Univariate Analyses $^{1}$} & \multicolumn{6}{|c|}{ Multivariate Analyses $^{1}$} \\
\hline & $\mathbf{n}$ & Median $^{2}$ & $\mathbf{H R}^{3}$ & $\mathbf{L C L}$ & UCL & $\mathbf{p}^{4}$ & $\mathbf{n}$ & Median $^{2}$ & $\mathbf{H R}^{3}$ & LCL & UCL & $\mathbf{p}^{4}$ \\
\hline \multicolumn{13}{|l|}{ Age (years) } \\
\hline$\leq 65$ & 183 & 41.7 & 1.00 & & & & 181 & 36.8 & 1.00 & & & \\
\hline$>65$ & 317 & 14.6 & 1.84 & 1.29 & 2.63 & .0008 & 317 & 15.9 & 1.67 & 1.16 & 2.40 & .006 \\
\hline \multicolumn{13}{|l|}{ Gender } \\
\hline Female & 95 & 21.2 & 1.00 & & & & 95 & 18.2 & 1.00 & & & \\
\hline Male & 405 & 22.1 & 0.98 & 0.66 & 1.46 & .92 & 403 & 22.6 & 0.88 & 0.59 & 1.32 & .54 \\
\hline \multicolumn{13}{|c|}{ Tumor Grade } \\
\hline Low & 219 & 50.5 & 1.00 & & & & 219 & 36.2 & 1.00 & & & \\
\hline High & 279 & 11.4 & 2.47 & 1.74 & 3.52 & $<.0001$ & 279 & 14.5 & 1.74 & 1.04 & 2.92 & .03 \\
\hline \multicolumn{13}{|l|}{ Tumor Stage } \\
\hline Ta or Tis & 253 & 44.6 & 1.00 & & & & 252 & 27.8 & 1.00 & & & \\
\hline $\mathrm{T} 1$ & 247 & 11.0 & 2.34 & 1.68 & 3.27 & $<.0001$ & 246 & 16.7 & 1.42 & 0.87 & 2.33 & .16 \\
\hline \multicolumn{13}{|c|}{ Lymph Nodes } \\
\hline $\mathrm{N} 0$ or $\mathrm{N}_{\mathrm{X}}$ & 487 & 23.5 & 1.00 & & & & 486 & 22.8 & 1.00 & & & \\
\hline N1 & 13 & 1.3 & 5.98 & 3.22 & 11.11 & $<.0001$ & 12 & 2.8 & 4.25 & 2.20 & 8.21 & $<.0001$ \\
\hline \multicolumn{13}{|l|}{ ANG } \\
\hline $0-2$ & 408 & 25.6 & 1.00 & & & & 408 & 24.3 & 1.00 & & & \\
\hline $3-4$ & 29 & 22.9 & 1.08 & 0.55 & 2.13 & .83 & 28 & 26.2 & 0.97 & 0.47 & 2.00 & .92 \\
\hline \multicolumn{13}{|l|}{ MMP-2 } \\
\hline $0-2$ & 175 & 26.1 & 1.00 & & & & 173 & 25.5 & 1.00 & & & \\
\hline $3-4$ & 281 & 21.6 & 1.06 & 0.75 & 1.49 & .75 & 281 & 20.4 & 1.09 & 0.77 & 1.55 & .63 \\
\hline $5-6$ & 2 & 17.8 & 1.12 & 0.56 & 2.22 & & 2 & 16.2 & 1.19 & 0.59 & 2.39 & \\
\hline \multicolumn{13}{|l|}{ p53 } \\
\hline $0-2$ & 284 & 29.8 & 1.00 & & & & 284 & 23.6 & 1.00 & & & \\
\hline $3-4$ & 173 & 18.3 & 1.33 & 1.01 & 1.75 & .04 & 173 & 21.5 & 1.08 & 0.81 & 1.44 & .60 \\
\hline $5-6$ & 24 & 11.3 & 1.77 & 1.03 & 3.06 & & 24 & 19.5 & 1.17 & 0.65 & 2.08 & \\
\hline \multicolumn{13}{|l|}{$\mathrm{Rb}$} \\
\hline $0-2$ & 97 & 23.6 & 1.00 & & & & 96 & 22.4 & 1.00 & & & \\
\hline $3-4$ & 347 & 27.4 & 0.93 & 0.62 & 1.40 & .74 & 347 & 28.0 & 0.89 & 0.58 & 1.35 & .58 \\
\hline $5-6$ & 3 & 31.7 & 0.87 & 0.38 & 1.97 & & 3 & 35.1 & 0.79 & 0.34 & 1.83 & \\
\hline \multicolumn{13}{|l|}{ PAI-1 } \\
\hline $0-2$ & 192 & 20.8 & 1.00 & & & & 191 & 17.3 & 1.00 & & & \\
\hline $3-4$ & 277 & 22.2 & 0.89 & 0.65 & 1.23 & .49 & 277 & 26.0 & 0.72 & 0.51 & 1.02 & .06 \\
\hline $5-6$ & 8 & 23.6 & 0.80 & 0.42 & 1.50 & & 8 & 39.2 & 0.52 & 0.26 & 1.03 & \\
\hline
\end{tabular}

${ }^{1}$ Univariate analyses are unadjusted. Multivariate analyses are adjusted for age, sex, tumor grade, tumor stage, and lymph nodes.

${ }^{2}$ Median is the median years progression free as estimated from a parametric model.

${ }^{3} \mathrm{HR}$ is the hazard ratio from a semiparametric (Cox proportional hazards) model. The 95\% confidence limits (LCL and UCL) are shown.

${ }^{4}$ The expression $\mathrm{p}$-values are for trend. 
Table 4b: Progression Free Results for Muscle Invasive Bladder Cancer

\begin{tabular}{|c|c|c|c|c|c|c|c|c|c|c|c|c|}
\hline & \multicolumn{6}{|c|}{ Univariate Analyses $^{1}$} & \multicolumn{6}{|c|}{ Multivariate Analyses $^{1}$} \\
\hline & $\mathbf{n}$ & Median $^{2}$ & $\mathbf{H R}^{3}$ & LCL & UCL & $\mathbf{p}^{4}$ & $\mathbf{n}$ & Median $^{2}$ & $\mathbf{H R}^{3}$ & LCL & $\mathbf{U C L}$ & $\mathbf{p}^{4}$ \\
\hline \multicolumn{13}{|l|}{ Age (years) } \\
\hline$\leq 65$ & 176 & 3.8 & 1.00 & & & & 176 & 3.5 & 1.00 & & & \\
\hline$>65$ & 248 & 2.4 & 1.30 & 1.04 & 1.63 & .02 & 248 & 2.4 & 1.26 & 1.00 & 1.58 & .05 \\
\hline \multicolumn{13}{|l|}{ Gender } \\
\hline Female & 130 & 2.0 & 1.00 & & & & 130 & 1.9 & 1.00 & & & \\
\hline Male & 297 & 3.4 & 0.71 & 0.56 & 0.89 & .003 & 294 & 3.4 & 0.68 & 0.54 & 0.86 & .001 \\
\hline \multicolumn{13}{|l|}{ Tumor Stage } \\
\hline $\mathrm{T} 2$ & 382 & 3.0 & 1.00 & & & & 381 & 2.9 & 1.00 & & & \\
\hline $\mathrm{T} 3$ or $\mathrm{T} 4$ & 44 & 2.6 & 1.06 & 0.75 & 1.49 & .76 & 43 & 2.5 & 1.07 & 0.75 & 1.53 & .72 \\
\hline \multicolumn{13}{|l|}{$\begin{array}{l}\text { Lymph } \\
\text { Nodes }\end{array}$} \\
\hline $\mathrm{N} 0$ or $\mathrm{N}_{\mathrm{X}}$ & 312 & 3.8 & 1.00 & & & & 309 & 3.8 & 1.00 & & & \\
\hline N1 & 115 & 1.3 & 1.99 & 1.56 & 2.54 & $<.0001$ & 115 & 1.3 & 2.01 & 1.57 & 2.57 & $<.0001$ \\
\hline \multicolumn{13}{|l|}{ ANG } \\
\hline $0-2$ & 318 & 3.1 & 1.00 & & & & 315 & 3.0 & 1.00 & & & \\
\hline $3-4$ & 35 & 1.5 & 1.43 & 0.97 & 2.11 & .07 & 35 & 1.5 & 1.40 & 0.95 & 2.07 & .09 \\
\hline \multicolumn{13}{|l|}{ MMP-2 } \\
\hline $0-2$ & 190 & 4.0 & 1.00 & & & & 189 & 3.9 & 1.00 & & & \\
\hline $3-4$ & 150 & 2.3 & 1.40 & 1.12 & 1.74 & .003 & 148 & 2.2 & 1.43 & 1.13 & 1.80 & .003 \\
\hline $5-6$ & 6 & 1.4 & 1.95 & 1.25 & 3.04 & & 6 & 1.3 & 2.03 & 1.28 & 3.22 & \\
\hline \multicolumn{13}{|l|}{ p53 } \\
\hline $0-2$ & 140 & 2.4 & 1.00 & & & & 140 & 2.5 & 1.00 & & & \\
\hline $3-4$ & 186 & 3.1 & 0.88 & 0.74 & 1.04 & .13 & 183 & 2.9 & 0.91 & 0.77 & 1.08 & .30 \\
\hline $5-6$ & 59 & 4.0 & 0.77 & 0.55 & 1.08 & & 59 & 3.5 & 0.83 & 0.59 & 1.17 & \\
\hline \multicolumn{13}{|l|}{$\mathrm{Rb}$} \\
\hline $0-2$ & 39 & 5.5 & 1.00 & & & & 38 & 5.6 & 1.00 & & & \\
\hline $3-4$ & 311 & 2.8 & 1.40 & 1.02 & 1.93 & .04 & 309 & 2.7 & 1.52 & 1.12 & 2.06 & .007 \\
\hline $5-6$ & 11 & 1.4 & 1.96 & 1.04 & 3.72 & & 11 & 1.3 & 2.31 & 1.26 & 4.23 & \\
\hline \multicolumn{13}{|l|}{ PAI-1 } \\
\hline $0-2$ & 143 & 3.9 & 1.00 & & & & 142 & 3.4 & 1.00 & & & \\
\hline $3-4$ & 228 & 2.5 & 1.29 & 1.04 & 1.58 & .02 & 226 & 2.6 & 1.19 & 0.96 & 1.48 & .11 \\
\hline $5-6$ & 16 & 1.6 & 1.66 & 1.09 & 2.51 & & 16 & 1.9 & 1.42 & 0.92 & 2.19 & \\
\hline
\end{tabular}

${ }^{1}$ Univariate analyses are unadjusted. Multivariate analyses are adjusted for age, sex, tumor stage, and lymph nodes.

${ }^{2}$ Median is the median years progression free as estimated from a parametric model.

${ }^{3} \mathrm{HR}$ is the hazard ratio from a semiparametric (Cox proportional hazards) model. The 95\% confidence limits (LCL and UCL) are shown.

${ }^{4}$ The expression p-values are for trend. 
influence MMP-2 gene, which may be a mechanism to promote $\mathrm{BCa}$ growth and angiogenesis. Interestingly in the current study, ANG expression was not associated with tumor grade or tumor stage. Perhaps the inability to probe for ANG was due to the condition of the paraffin embedded tissue (i.e., older sections may have some degradation of proteins). Thus, more research is needed to confirm ANG's presence in human $\mathrm{BCa}$. On the other hand, MMP-2 expression was greater in low-grade and low-stage $\mathrm{BCa}$ and was associated with a reduction in recurrence-free survival as well as reduction in overall survival.

PAI-1 is the primary inhibitor of tissue-type plasminogen activator (tPA) and urokinase-type plasminogen activator (uPA), and it acts to suppress tissue and plasma fibrinolysis via plasmin conversion [38].

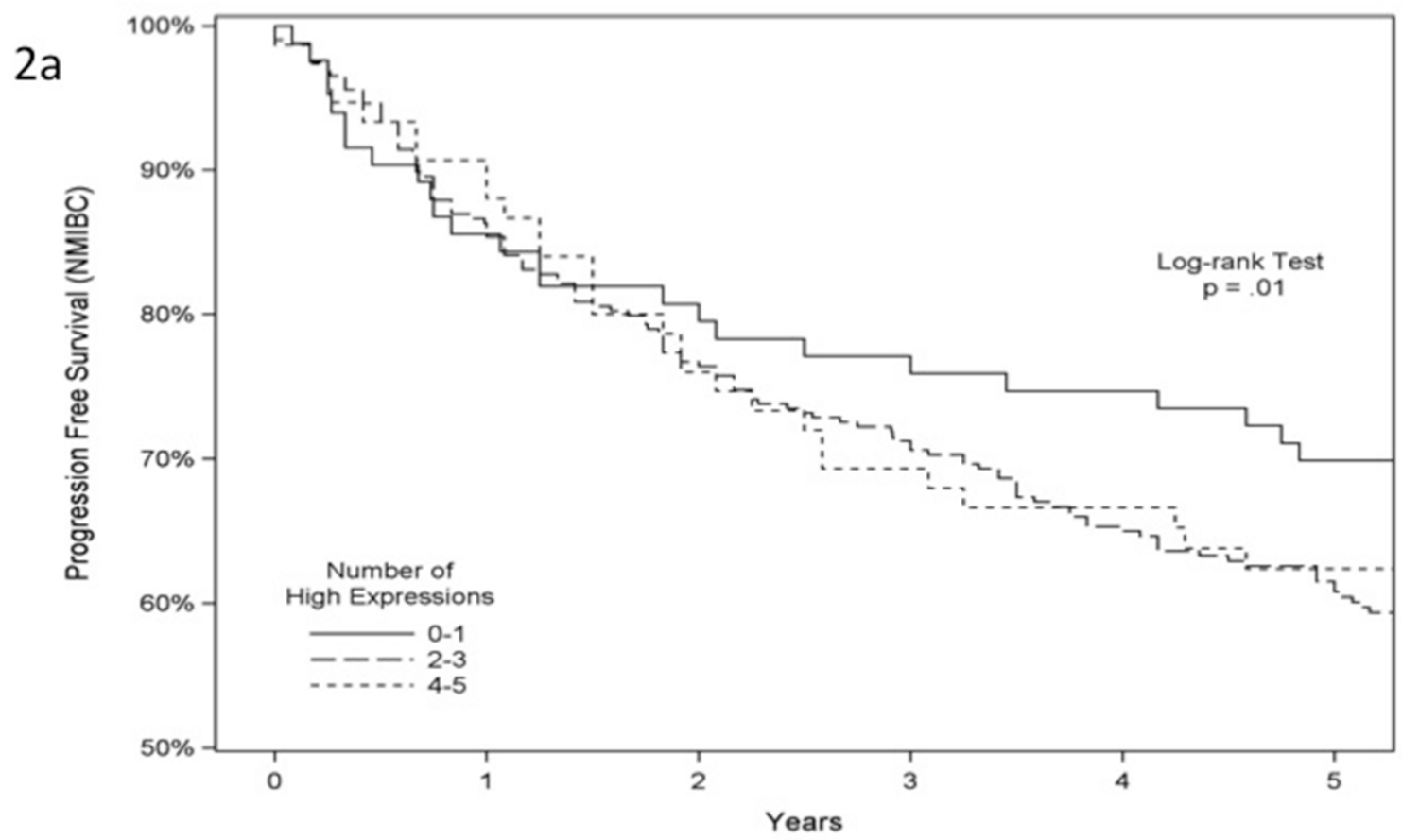

$2 b$

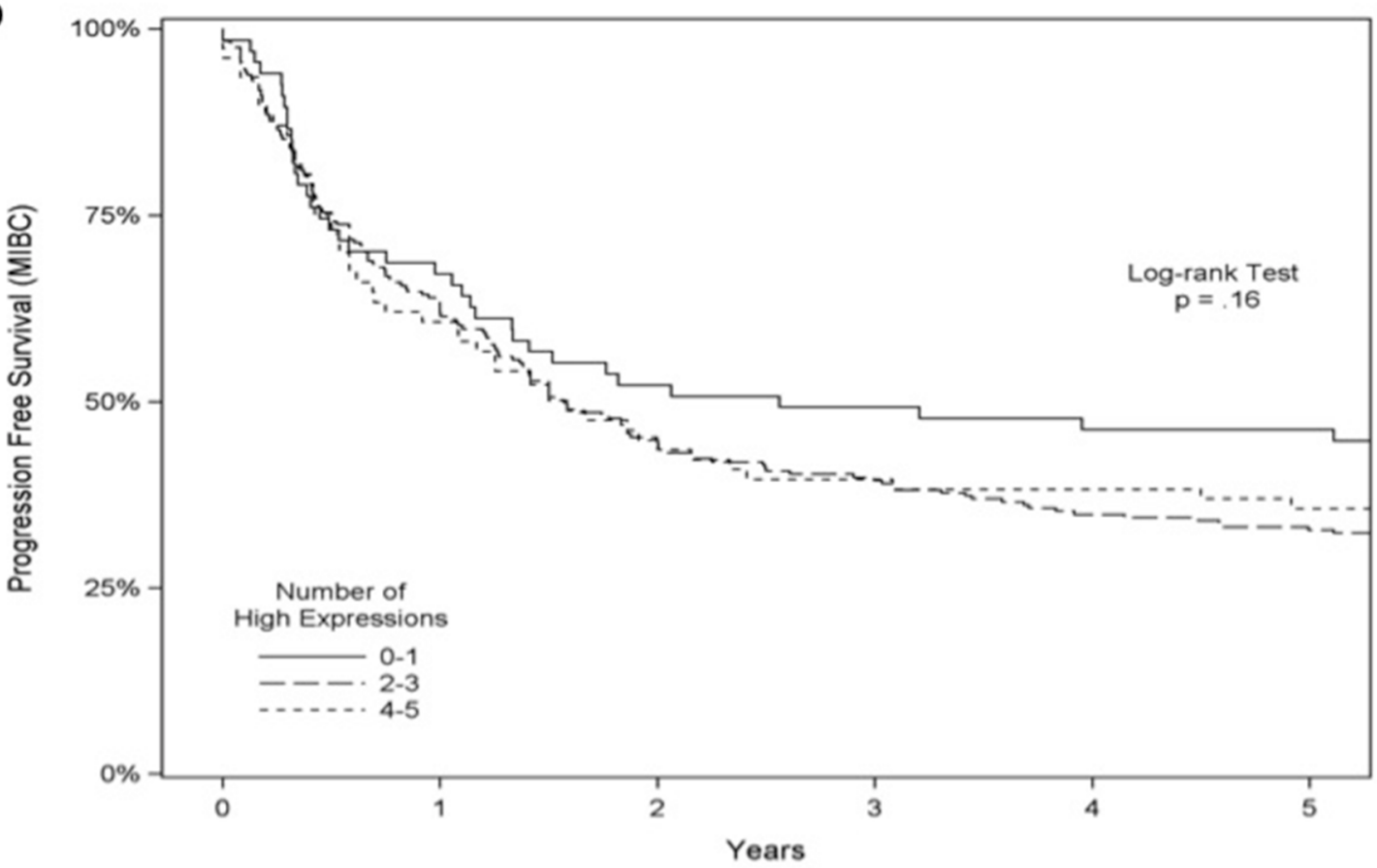

Figure 2: Progression-free survival analysis of 939 patients with bladder cancer. Progression-free survival according to immunostaining status of ANG, MMP-2, p53, RB and PAI-1 in NMIBC (A) and MIBC (B). 
Table 5a: Overall Survival Results for Non-Muscle Invasive Bladder Cancer

\begin{tabular}{|c|c|c|c|c|c|c|c|c|c|c|c|c|}
\hline & \multicolumn{6}{|c|}{ Univariate Analyses $^{1}$} & \multicolumn{6}{|c|}{ Multivariate Analyses $^{1}$} \\
\hline & $\bar{n}$ & Median $^{2}$ & $\mathrm{HR}^{3}$ & LCL & UCL & $\mathbf{p}^{4}$ & $\mathbf{n}$ & Median $^{2}$ & $\mathrm{HR}^{3}$ & LCL & UCL & $\mathbf{p}^{4}$ \\
\hline \multicolumn{13}{|l|}{ Age (years) } \\
\hline$\leq 65$ & 183 & 16.7 & 1.00 & & & & 181 & 15.4 & 1.00 & & & \\
\hline$>65$ & 317 & 7.4 & 2.59 & 1.93 & 3.48 & $<.0001$ & 317 & 7.5 & 2.41 & 1.78 & 3.26 & $<.0001$ \\
\hline \multicolumn{13}{|l|}{ Gender } \\
\hline Female & 95 & 10.8 & 1.00 & & & & 95 & 10.2 & 1.00 & & & \\
\hline Male & 405 & 9.6 & 1.14 & 0.82 & 1.58 & .44 & 403 & 9.7 & 1.07 & 0.77 & 1.49 & .68 \\
\hline \multicolumn{13}{|c|}{ Tumor Grade } \\
\hline Low & 219 & 14.2 & 1.00 & & & & 219 & 14.0 & 1.00 & & & \\
\hline High & 279 & 7.2 & 2.10 & 1.62 & 2.73 & $<.0001$ & 279 & 7.4 & 2.11 & 1.43 & 3.12 & .0002 \\
\hline \multicolumn{13}{|l|}{ Tumor Stage } \\
\hline Ta or Tis & 253 & 12.4 & 1.00 & & & & 252 & 9.3 & 1.00 & & & \\
\hline $\mathrm{T} 1$ & 247 & 7.7 & 1.67 & 1.30 & 2.15 & .0001 & 246 & 10.3 & 0.88 & 0.61 & 1.27 & .50 \\
\hline \multicolumn{13}{|c|}{ Lymph Nodes } \\
\hline N0 or $\mathrm{N}_{\mathrm{X}}$ & 487 & 10.2 & 1.00 & & & & 486 & 10.0 & 1.00 & & & \\
\hline N1 & 13 & 2.7 & 4.03 & 2.19 & 7.41 & $<.0001$ & 12 & 4.3 & 2.66 & 1.39 & 5.08 & .003 \\
\hline \multicolumn{13}{|l|}{ ANG } \\
\hline $0-2$ & 408 & 9.6 & 1.00 & & & & 408 & 9.5 & 1.00 & & & \\
\hline $3-4$ & 29 & 12.6 & 0.75 & 0.42 & 1.35 & .34 & 28 & 12.8 & 0.71 & 0.39 & 1.31 & .28 \\
\hline \multicolumn{13}{|l|}{ MMP-2 } \\
\hline $0-2$ & 175 & 12.4 & 1.00 & & & & 173 & 11.9 & 1.00 & & & \\
\hline $3-4$ & 281 & 9.1 & 1.40 & 1.06 & 1.84 & .02 & 281 & 9.1 & 1.37 & 1.04 & 1.81 & .03 \\
\hline $5-6$ & 2 & 6.6 & 1.95 & 1.12 & 3.37 & & 2 & 7.0 & 1.87 & 1.08 & 3.26 & \\
\hline \multicolumn{13}{|l|}{ p53 } \\
\hline $0-2$ & 284 & 10.4 & 1.00 & & & & 284 & 9.5 & 1.00 & & & \\
\hline $3-4$ & 173 & 9.7 & 1.08 & 0.87 & 1.35 & .49 & 173 & 10.6 & 0.89 & 0.70 & 1.13 & .34 \\
\hline $5-6$ & 24 & 9.1 & 1.17 & 0.75 & 1.82 & & 24 & 11.7 & 0.80 & 0.49 & 1.28 & \\
\hline \multicolumn{13}{|l|}{$\mathrm{Rb}$} \\
\hline $0-2$ & 114 & 17.9 & 1.00 & & & & 114 & 14.7 & 1.00 & & & \\
\hline $3-4$ & 313 & 8.7 & 2.25 & 1.72 & 2.95 & $<.0001$ & 312 & 9.2 & 1.76 & 1.34 & 2.31 & .0001 \\
\hline $5-6$ & 20 & 4.2 & 5.07 & 2.95 & 8.72 & & 20 & 5.8 & 3.08 & 1.79 & 5.32 & \\
\hline \multicolumn{13}{|l|}{ PAI-1 } \\
\hline $0-2$ & 192 & 13.3 & 1.00 & & & & 191 & 12.0 & 1.00 & & & \\
\hline $3-4$ & 277 & 7.5 & 1.90 & 1.48 & 2.43 & $<.0001$ & 277 & 8.1 & 1.61 & 1.23 & 2.09 & .0004 \\
\hline $5-6$ & 8 & 4.3 & 3.60 & 2.20 & 5.89 & & 8 & 5.5 & 2.58 & 1.52 & 4.38 & \\
\hline
\end{tabular}

${ }^{1}$ Univariate analyses are unadjusted. Multivariate analyses are adjusted for age, sex, tumor grade, tumor stage, and lymph nodes.

${ }^{2}$ Median is the median years overall survival as estimated from a parametric model.

${ }^{3} \mathrm{HR}$ is the hazard ratio from a semiparametric (Cox proportional hazards) model. The 95\% confidence limits (LCL and UCL) are shown.

${ }^{4}$ The expression $\mathrm{p}$-values are for trend. 
Table 5b: Overall Survival Results for Muscle Invasive Bladder Cancer

\begin{tabular}{|c|c|c|c|c|c|c|c|c|c|c|c|c|}
\hline & \multicolumn{6}{|c|}{ Univariate Analyses $^{1}$} & \multicolumn{6}{|c|}{ Multivariate Analyses ${ }^{1}$} \\
\hline & $\mathbf{n}$ & Median $^{2}$ & $\mathbf{H R}^{3}$ & LCL & UCL & $\mathbf{p}^{4}$ & $\mathbf{n}$ & Median $^{2}$ & $\mathbf{H R}^{3}$ & LCL & UCL & $\mathbf{p}^{4}$ \\
\hline \multicolumn{13}{|l|}{ Age (years) } \\
\hline$\leq 65$ & 176 & 5.3 & 1.00 & & & & 176 & 5.1 & 1.00 & & & \\
\hline$>65$ & 248 & 3.3 & 1.41 & 1.12 & 1.77 & .004 & 248 & 3.4 & 1.38 & 1.10 & 1.74 & .006 \\
\hline \multicolumn{13}{|l|}{ Gender } \\
\hline Female & 130 & 2.8 & 1.00 & & & & 130 & 2.7 & 1.00 & & & \\
\hline Male & 297 & 4.7 & 0.70 & 0.55 & 0.88 & .002 & 294 & 4.7 & 0.68 & 0.54 & 0.86 & .002 \\
\hline \multicolumn{13}{|l|}{ Tumor Stage } \\
\hline $\mathrm{T} 2$ & 382 & 4.1 & 1.00 & & & & 381 & 4.1 & 1.00 & & & \\
\hline $\mathrm{T} 3$ or $\mathrm{T} 4$ & 44 & 3.7 & 1.07 & 0.75 & 1.52 & .73 & 43 & 3.5 & 1.11 & 0.76 & 1.60 & .59 \\
\hline \multicolumn{13}{|c|}{ Lymph Nodes } \\
\hline N0 or $\mathrm{N}_{\mathrm{X}}$ & 312 & 4.6 & 1.00 & & & & 309 & 4.6 & 1.00 & & & \\
\hline N1 & 115 & 2.7 & 1.40 & 1.09 & 1.81 & .008 & 115 & 2.7 & 1.40 & 1.09 & 1.80 & .009 \\
\hline \multicolumn{13}{|l|}{ ANG } \\
\hline $0-2$ & 318 & 4.1 & 1.00 & & & & 315 & 4.0 & 1.00 & & & \\
\hline $3-4$ & 35 & 2.8 & 1.26 & 0.84 & 1.88 & .26 & 35 & 3.1 & 1.20 & 0.80 & 1.80 & .37 \\
\hline \multicolumn{13}{|l|}{ MMP-2 } \\
\hline $0-2$ & 190 & 5.5 & 1.00 & & & & 189 & 5.4 & 1.00 & & & \\
\hline $3-4$ & 150 & 3.2 & 1.48 & 1.18 & 1.86 & .0008 & 148 & 3.3 & 1.44 & 1.14 & 1.83 & .003 \\
\hline $5-6$ & 6 & 1.9 & 2.19 & 1.38 & 3.46 & & 6 & 2.0 & 2.08 & 1.29 & 3.33 & \\
\hline \multicolumn{13}{|l|}{ p53 } \\
\hline $0-2$ & 140 & 3.2 & 1.00 & & & & 140 & 3.3 & 1.00 & & & \\
\hline $3-4$ & 186 & 4.2 & 0.84 & 0.71 & 1.00 & .05 & 183 & 4.2 & 0.86 & 0.73 & 1.02 & .09 \\
\hline $5-6$ & 59 & 5.6 & 0.70 & 0.50 & 0.99 & & 59 & 5.2 & 0.74 & 0.53 & 1.05 & \\
\hline \multicolumn{13}{|l|}{$\mathrm{Rb}$} \\
\hline $0-2$ & 39 & 6.5 & 1.00 & & & & 38 & 6.6 & 1.00 & & & \\
\hline $3-4$ & 311 & 3.7 & 1.43 & 1.03 & 2.00 & .03 & 309 & 3.7 & 1.48 & 1.07 & 2.03 & .02 \\
\hline $5-6$ & 11 & 2.1 & 2.06 & 1.06 & 4.00 & & 11 & 2.1 & 2.18 & 1.15 & 4.13 & \\
\hline \multicolumn{13}{|l|}{ PAI-1 } \\
\hline $0-2$ & 143 & 5.3 & 1.00 & & & & 142 & 5.0 & 1.00 & & & \\
\hline $3-4$ & 228 & 3.5 & 1.37 & 1.11 & 1.70 & .003 & 226 & 3.5 & 1.30 & 1.05 & 1.62 & .02 \\
\hline $5-6$ & 16 & 2.3 & 1.88 & 1.23 & 2.88 & & 16 & 2.5 & 1.69 & 1.09 & 2.62 & \\
\hline
\end{tabular}

${ }^{1}$ Univariate analyses are unadjusted. Multivariate analyses are adjusted for age, sex, tumor stage, and lymph nodes.

${ }^{2}$ Median is the median years overall survival as estimated from a parametric model.

${ }^{3} \mathrm{HR}$ is the hazard ratio from a semiparametric (Cox proportional hazards) model. The 95\% confidence limits (LCL and UCL) are shown.

${ }^{4}$ The expression $\mathrm{p}$-values are for trend. 
We found that the down-regulation of PAI-1 led to a) an inhibition in cell proliferation and $b$ ) a potent arrest in the $\mathrm{G}_{1}$ to $\mathrm{S}$ phase of the cell cycle, a phenomena associated with reduction in cyclin D3/cdk4/6 and cyclin E/cdk2 and an increase in cell cycle inhibitors, p53, p $21^{\text {Cip1/Waf1, }}$ and $\mathrm{p} 27^{\mathrm{kip} 1}$ [18]. Little attention has been given to PAI1 in the human urinary bladder. For example, only two groups have reported PAI-1 levels for BCa patients. Urquidi et al. noted a significant increase in urothelial cell PAI-1 levels in patients bearing bladder tumors compared to non-tumor bearing patients [39]. Becker et al. reported significantly higher PAI-1 levels in tissue and plasma samples, but not in urine, from patients with $\mathrm{BCa}$ compared with controls [40]. Although the function of PAI-1 is complex, further investigation is warranted in the hope that it can provide insight into specific aspects of tumor biology and the identification of tumor cell vulnerabilities for therapeutic exploitation. Interestingly in the current study, higher PAI-1 expression was associated with high-grade compared to low-grade, while higher
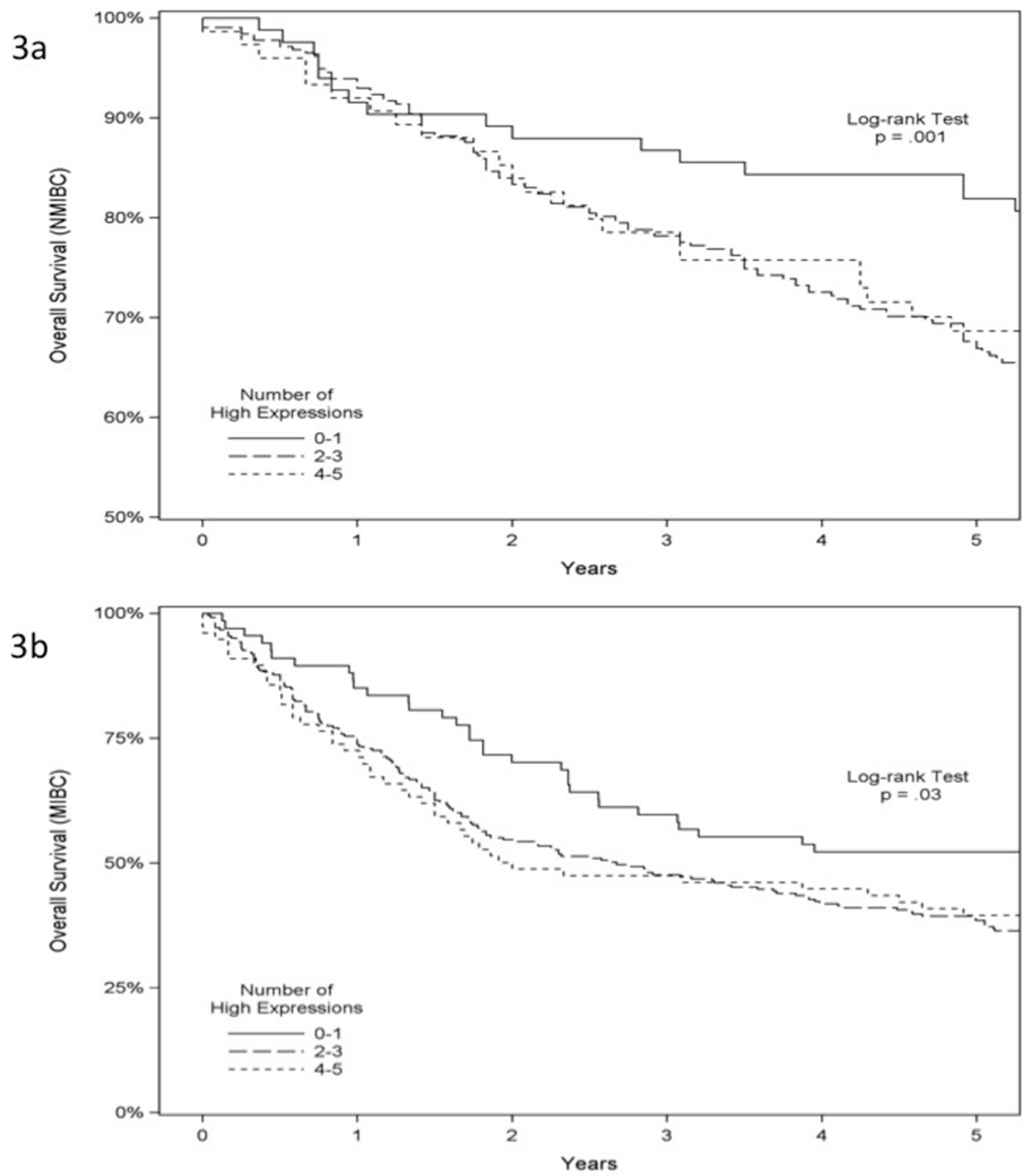

Figure 3: Overall survival analysis of 939 patients with bladder cancer. Overall survival according to immunostaining status of ANG, MMP-2, p53, RB and PAI-1 in NMIBC (A) and MIBC (B). 
expression of p53 and RB were associated with highgrade and high stage disease. High PAI-1, p53 and RB were associated with a reduction in survival. Perhaps, the ability to demonstrate the difference in PAI-1 expression in this cohort compared to our previous cohort is related to the increase in the sample sizes of the current study. The high expression levels of p53 and RB have been well documented in $\mathrm{BCa}[41,42]$. In fact several studies have reported on the prognostic significance of p53 expression in bladder tumors. For example, Cordon-Cardo and others, noted an association between the p53-positive phenotype and disease progression $(\mathrm{p}<0.001)$, as well as reduced survival $(p<0.001)$ in a small cohort comprised of 29 NMIBC [43]. Similarly in MIBC, Shariat and others, reported that alteration in $\mathrm{p} 53$ (or p21, pRB, p16) was independently associated with disease progression ( $\mathrm{p}$ $\leq 0.038)$ and disease-specific survival $(p<0.039)$ in a small cohort of 127 MIBC treated with cystectomy [44]. Differences in outcomes associated with these studies and the current study may be related to our large, diverse cohort of 939 subjects. In the only phase III randomized study assessing the prognostic significance of p53 in $\mathrm{BCa}$, Stadler and others, could not validate the prognostic significance of p53 in 521 patients undergoing adjuvant chemotherapy [45]. However, the current report begins to elucidate a specific pathway involving PAI-1, p53 and $\mathrm{RB}$ in human $\mathrm{BCa}$. On the other hand, MMP-2 expression was greater in low-grade and low-stage $\mathrm{BCa}$ and was associated with a reduction in recurrence-free survival, as well as a reduction in overall survival.

Our findings in this report also have therapeutic implications regarding the potential utility of targeting these proteins directly. We and others have previously reported that blocking ANG, MMP-2, p53, RB or PAI1 in bladder cell lines can significantly reduce cell proliferation/survival $[17,18]$, migration and invasion $[18,45,46]$ and angiogenesis [17, 47-49]. Our current results, when combined with these previous observations, further strengthen the rationale of targeting MMP-2, RB and PAI- 1 directly for treatment of BCa.

In summary, we have demonstrated in this study that MMP-2, RB and PAI-1 expression is correlated with poor prognosis of patients with $\mathrm{BCa}$. Our findings support the hypothesis that targeting these three molecules may improve the survival of patients with $\mathrm{BCa}$.

\section{MATERIALS AND METHODS}

\section{Patients and clinicopathologic information}

The study was performed after approval by the Western Institutional Review Board under a request of waiver of consent on archived pathologic specimens. Tissue microarrays associated with two independent patient cohorts were used in the current study. Cohort \#1 was comprised of 587 patients diagnosed with
BCa from 1979-2007 at Aarhus University Hospital (Aarhus, Denmark) [21], including 2 sub-cohorts; one with 223 primary $\mathrm{Ta} / \mathrm{T} 1$ tumors from patients treated by transurethral resection of the bladder and one with 364 tumors from radical cystectomy patients. Cohort \#2 was comprised of 352 patients diagnosed with BCa from 19842005 at Uppsala University Hospital (Uppsala, Sweden) [50]. Thus, samples from 939 patients were available for analysis. Demographic, clinical, disease and treatment characteristics are presented in Table 1. The median follow-up for the entire cohort was 5.37 years.

Follow-up data also included progression-free survival (PFS), cancer-specific survival (CSS) and overall survival (OS). The endpoints of recurrence-free survival and OS were calculated from the date of surgery to the date of the recurrence or last follow-up.

\section{Immunohistochemcal staining of tissue microarrays}

Immunostaining was performed using standard protocols. TMAs were deparaffinized in xylene and rehydrated using graded percentages of ethanol followed by antigen retrieval with citric acid buffer $\left(\mathrm{pH} 6.0,95^{\circ} \mathrm{C}\right.$ for $20 \mathrm{~min}$ ). The slides were treated with $3 \%$ hydrogen peroxide in water to block endogenous peroxidase activity. Staining for ANG, MMP-2, p53, RB and PAI-1 was conducted using mouse anti-human ANG antibody (C-1; 1:25 dilution in blocking buffer, Santa Cruz Biotechnology), rabbit anti-MMP-2 antibody (AB19167; 1:100 dilution in Biocare Background Sniper, EMD Millipore), mouse anti-p53 antibody (NCL-L-p53-DO7; 1:100 dilution in Biocare Background Sniper, Leica Biosystems), rabbit anti-Rb Antibody (C-15; 1:500 dilution in Biocare Background Sniper, Santa Cruz Biotechnology), and rabbit anti-PAI-1 antibody (HPA050039; 1:100 dilution in blocking buffer, Sigma-Aldrich), respectively. The antibodies' specificity information can be found in the manufacturers' datasheets or our previous publication [20]. Biotin-labeled horse anti-mouse IgG $(2 \mu \mathrm{g} / \mathrm{ml}$ in blocking buffer, Vector Laboratories) was used as the secondary antibody. Immunoreactive signals were amplified by the formation of avidin-biotin peroxidase complexes and visualized using 3, 3'- diaminobenzidine (DAB). Nuclear counterstaining was conducted with hematoxylin.

The expression levels of ANG, MMP-2, p53, RB and PAI-1 were scored by assigning a proportion score and an intensity score $[17,18,51,52]$. The estimated proportion $(0=0 \%$ of cells; $1=1 \%$ to $40 \% ; 2=41 \%$ to $75 \%$ and $3=76 \%$ to $100 \%$ ) and the average intensity $(0=$ none; 1 = weak; 2 = intermediate and $3=$ strong $)$ of immunoreactive tumor cells were assessed. The proportion and intensity scores were combined to obtain a total staining score for each protein, which ranged from 0 to 6 . Thus, the protein expression levels were determined based on the total ANG, MMP-2, p53, RB and PAI-1 staining 
score as follows: none $=0$, low $=1$ or 2 , moderate $=3$ or 4 , high $=5$ or 6 . Two investigators (OTMC, YS), who were blinded to the clinicopathologic data and clinical outcomes, scored each core. When there was a discrepancy in the scoring, a third investigator reviewed consensus scoring was obtained.

Human lung (SERPINE1, MMP-2), liver (ANG) and tonsil (p53, Rb) were used as positive controls, and omitting the primary antibody served as the negative controls.

\section{Statistical analysis}

SAS 9.4 (Cary, NC) was used to perform the statistical analyses. The relationship between immunoexpression of the five targets and clinicopathological features were assessed with the Fisher's exact test (crosstabs), log-rank tests (progressionfree survival, cancer-specific survival and overall survival), and multivariate Cox proportional hazards regression (used to adjust for demographic and clinical parameters). All tests were 2-tailed.

\section{Abbreviations}

BCa: bladder cancer; NMIBC: non muscle invasive bladder cancer; MIBC: muscle invasive bladder cancer; ANG - angiogenin; PAI-1: plasminogen activator inhibitor 1; TMA: tissue microarray; HR: hazard ratio; OS: overall survival; tPA: tissue plasminogen activator; uPA: urokinase plasminogen activator; CSS: cancer specific survival; PFS: progression free survival; DAB: 3, 3'diaminobenzidine.

\section{Author contributions}

Owen T.M. Chan: performed IHC, assisted in interpreting IHC and drafted manuscript.

Hideki Furuya: participated in study design and helped to draft the manuscript.

Ian Pagano: organized and analyzed data.

Yoshiko Shimizu: assisted with IHC, organized data.

Kanani Hokutan: assisted with IHC, organized data.

Lars Dyrskjøt: participated in study design, provided

TMA and helped to draft the manuscript.

Jørgen Bjerggaard Jensen: participated in study design and helped to draft the manuscript.

Per-Uno Malmstrom: participated in study design, provided TMA and helped to draft the manuscript.

Ulrika Segersten: participated in study design and helped to draft the manuscript.

Filip Janku: participated in study design and helped to draft the manuscript.

Charles J. Rosser: conceived the study, and participated in its design and coordination and helped to draft the manuscript.

\section{ACKNOWLEDGMENTS}

We thank M.D. Anderson Science Park Histology and Tissue Processing core facility and the University of Hawaii Cancer Center Pathology Shared Resource for their support.

\section{CONFLICTS OF INTEREST}

All authors declare that they have no competing interests.

\section{FUNDING}

This work was supported by research grants from Weinman Foundation Fund (PI-CJR), 5P30CA0717890-6071 (CJR Investigator) and 1 R01 CA198887 01A (CJR).

\section{REFERENCES}

1. Siegel RL, Miller KD, Jemal A. Cancer statistics, 2016. CA Cancer J Clin. 2016; 66: 7-30. doi: 10.3322/caac.21332.

2. Aben KK, Kiemeney LA. Epidemiology of bladder cancer. Eur Urol. 1999; 36: 660-72.

3. Hall MC, Chang SS, Dalbagni G, Pruthi RS, Seigne JD, Skinner EC, Wolf JS Jr, Schellhammer PF. Guideline for the management of nonmuscle invasive bladder cancer (stages Ta, T1, and Tis): 2007 update. J Urol. 2007; 178: 2314-30. doi: 10.1016/j.juro.2007.09.003.

4. Babjuk M, Oosterlinck W, Sylvester R, Kaasinen E, Bohle A, Palou-Redorta J, European Association of U. EAU guidelines on non-muscle-invasive urothelial carcinoma of the bladder. Eur Urol. 2008; 54: 303-14. doi: 10.1016/j. eururo.2008.04.051.

5. Surveillance, Epidemiology, and End Results Program.

6. Stenzl A, Cowan NC, De Santis M, Kuczyk MA, Merseburger AS, Ribal MJ, Sherif A, Witjes JA, European Association of U. Treatment of muscle-invasive and metastatic bladder cancer: update of the EAU guidelines. Eur Urol. 2011; 59: 1009-18. doi: 10.1016/j. eururo.2011.03.023.

7. Sternberg CN, Donat SM, Bellmunt J, Millikan RE, Stadler W, De Mulder P, Sherif A, von der Maase H, Tsukamoto T, Soloway MS. Chemotherapy for bladder cancer: treatment guidelines for neoadjuvant chemotherapy, bladder preservation, adjuvant chemotherapy, and metastatic cancer. Urology. 2007; 69: 62-79. doi: 10.1016/j. urology.2006.10.041.

8. Yang N, Feng S, Shedden K, Xie X, Liu Y, Rosser CJ, Lubman DM, Goodison S. Urinary glycoprotein biomarker discovery for bladder cancer detection using LC/MS-MS and label-free quantification. Clin Cancer Res. 2011; 17: 3349-59. doi: 10.1158/1078-0432.CCR-10-3121. 
9. Kreunin P, Zhao J, Rosser C, Urquidi V, Lubman DM, Goodison S. Bladder cancer associated glycoprotein signatures revealed by urinary proteomic profiling. J Proteome Res. 2007; 6: 2631-9. doi: 10.1021/pr0700807.

10. Urquidi V, Goodison S, Cai Y, Sun Y, Rosser CJ. A candidate molecular biomarker panel for the detection of bladder cancer. Cancer Epidemiol Biomarkers Prev. 2012; 21: 2149-58. doi: 10.1158/1055-9965.EPI-12-0428.

11. Rosser CJ, Liu L, Sun Y, Villicana P, McCullers M, Porvasnik S, Young PR, Parker AS, Goodison S. Bladder cancer-associated gene expression signatures identified by profiling of exfoliated urothelia. Cancer Epidemiol Biomarkers Prev. 2009; 18: 444-53. doi: 10.1158/10559965.EPI-08-1002.

12. Goodison S, Chang M, Dai Y, Urquidi V, Rosser CJ. A multi-analyte assay for the non-invasive detection of bladder cancer. PLoS One. 2012; 7: e47469. doi: 10.1371/ journal.pone.0047469.

13. Rosser CJ, Ross S, Chang M, Dai Y, Mengual L, Zhang G, Kim J, Urquidi V, Alcaraz A, Goodison S. Multiplex protein signature for the detection of bladder cancer in voided urine samples. J Urol. 2013; 190: 2257-62. doi: 10.1016/j. juro.2013.06.011.

14. Chen LM, Chang M, Dai Y, Chai KX, Dyrskjot L, SanchezCarbayo M, Szarvas T, Zwarthoff EC, Lokeshwar V, Jeronimo C, Parker AS, Ross S, Borre M, et al. External validation of a multiplex urinary protein panel for the detection of bladder cancer in a multicenter cohort. Cancer Epidemiol Biomarkers Prev. 2014; 23: 1804-12. doi: 10.1158/1055-9965.EPI-14-0029.

15. Rosser CJ, Chang M, Dai Y, Ross S, Mengual L, Alcaraz A, Goodison S. Urinary protein biomarker panel for the detection of recurrent bladder cancer. Cancer Epidemiol Biomarkers Prev. 2014; 23: 1340-5. doi: 10.1158/10559965.EPI-14-0035.

16. Shimizu Y, Furuya H, Bryant Greenwood P, Chan O, Dai Y, Thornquist MD, Goodison S, Rosser CJ. A multiplex immunoassay for the non-invasive detection of bladder cancer. J Transl Med. 2016; 14: 31. doi: 10.1186/ s12967-016-0783-2.

17. Miyake M, Goodison S, Lawton A, Gomes-Giacoia E, Rosser CJ. Angiogenin promotes tumoral growth and angiogenesis by regulating matrix metallopeptidase-2 expression via the ERK1/2 pathway. Oncogene. 2015; 34: 890-901. doi: 10.1038/onc.2014.2.

18. Gomes Giacoia E, Miyake M, Lawton A, Goodison S, Rosser CJ. PAI-1 Leads to G1-phase Cell Cycle Progression through Cyclin D3/CDK4/6 Up-regulation. Mol Cancer Res. 2014; 12: 322-34. doi: 10.1158/15417786.MCR-13-0543.

19. Gomes-Giacoia E, Miyake M, Goodison S, Rosser CJ. Targeting plasminogen activator inhibitor-1 inhibits angiogenesis and tumor growth in a human cancer xenograft model. Mol Cancer Ther. 2013; 12: 2697-708. doi: 10.1158/1535-7163.MCT-13-0500.
20. Zhang G, Gomes-Giacoia E, Dai Y, Lawton A, Miyake M, Furuya H, Goodison S, Rosser CJ. Validation and clinicopathologic associations of a urine-based bladder cancer biomarker signature. Diagn Pathol. 2014; 9: 200. doi: 10.1186/s13000-014-0200-1.

21. Jensen JB, Munksgaard PP, Sorensen CM, Fristrup N, Birkenkamp-Demtroder K, Ulhoi BP, Jensen KM, Orntoft TF, Dyrskjot L. High expression of karyopherin-alpha2 defines poor prognosis in non-muscle-invasive bladder cancer and in patients with invasive bladder cancer undergoing radical cystectomy. Eur Urol. 2011; 59: 841-8. doi: 10.1016/j.eururo.2011.01.048.

22. Fett JW, Strydom DJ, Lobb RR, Alderman EM, Bethune JL, Riordan JF, Vallee BL. Isolation and characterization of angiogenin, an angiogenic protein from human carcinoma cells. Biochemistry. 1985; 24: 5480-6.

23. Nilsson UW, Abrahamsson A, Dabrosin C. Angiogenin regulation by estradiol in breast tissue: tamoxifen inhibits angiogenin nuclear translocation and antiangiogenin therapy reduces breast cancer growth in vivo. Clin Cancer Res. 2010; 16: 3659-69. doi: 10.1158/1078-0432.CCR-10-0501.

24. Hisai H, Kato J, Kobune M, Murakami T, Miyanishi K, Takahashi M, Yoshizaki N, Takimoto R, Terui T, Niitsu Y. Increased expression of angiogenin in hepatocellular carcinoma in correlation with tumor vascularity. Clin Cancer Res. 2003; 9: 4852-9.

25. Geis $\mathrm{T}$, Doring $\mathrm{C}$, Popp R, Grossmann N, Fleming I, Hansmann ML, Dehne N, Brune B. HIF-2alphadependent PAI-1 induction contributes to angiogenesis in hepatocellular carcinoma. Exp Cell Res. 2015; 331: 46-57. doi: 10.1016/j.yexcr.2014.11.018.

26. Masuda T, Hattori N, Senoo T, Akita S, Ishikawa N, Fujitaka K, Haruta Y, Murai H, Kohno N. SK-216, an inhibitor of plasminogen activator inhibitor-1, limits tumor progression and angiogenesis. Mol Cancer Ther. 2013; 12: 2378-88. doi: 10.1158/1535-7163.MCT-13-0041.

27. Morgan H, Hill PA. Human breast cancer cell-mediated bone collagen degradation requires plasminogen activation and matrix metalloproteinase activity. Cancer Cell Int. 2005; 5: 1. doi: 10.1186/1475-2867-5-1.

28. Barcena C, Stefanovic M, Tutusaus A, Martinez-Nieto GA, Martinez L, Garcia-Ruiz C, de Mingo A, Caballeria J, Fernandez-Checa JC, Mari M, Morales A. Angiogenin secretion from hepatoma cells activates hepatic stellate cells to amplify a self-sustained cycle promoting liver cancer. Sci Rep. 2015; 5: 7916. doi: 10.1038/srep07916.

29. Blood $\mathrm{CH}$, Zetter BR. Tumor interactions with the vasculature: angiogenesis and tumor metastasis. Biochim Biophys Acta. 1990; 1032: 89-118.

30. Schneider-Merck T, Pohnke Y, Kempf R, Christian M, Brosens JJ, Gellersen B. Physical interaction and mutual transrepression between CCAAT/enhancer-binding protein beta and the p53 tumor suppressor. J Biol Chem. 2006; 281: 269-78. doi: 10.1074/jbc.M503459200. 
31. Ho JS, Ma W, Mao DY, Benchimol S. p53-Dependent transcriptional repression of c-myc is required for $\mathrm{G} 1$ cell cycle arrest. Mol Cell Biol. 2005; 25: 7423-31. doi: 10.1128/MCB.25.17.7423-7431.2005.

32. Scian MJ, Frum R, Deb SP, Deb S. Transactivation and transrepression studies with p53. Methods Mol Biol. 2003; 234: 93-110. doi: 10.1385/1-59259-408-5:93.

33. Liao CC, Tsai CY, Chang WC, Lee WH, Wang JM. RB.E2F1 complex mediates DNA damage responses through transcriptional regulation of ZBRK1. J Biol Chem. 2010; 285: 33134-43. doi: 10.1074/jbc.M110.143461.

34. Meier K, Brehm A. Chromatin regulation: how complex does it get? Epigenetics. 2014; 9: 1485-95. doi: 10.4161/15592294.2014.971580.

35. Scott MC, Sarver AL, Tomiyasu H, Cornax I, Van Etten J, Varshney J, O'Sullivan MG, Subramanian S, Modiano JF. Aberrant Retinoblastoma (RB)-E2F Transcriptional Regulation Defines Molecular Phenotypes of Osteosarcoma. J Biol Chem. 2015; 290: 28070-83. doi: 10.1074/jbc. M115.679696.

36. Peres R, Furuya H, Pagano I, Shimizu Y, Hokutan K, Rosser CJ. Angiogenin contributes to bladder cancer tumorigenesis by DNMT3b-mediated MMP2 activation. Oncotarget. 2016; 7: 43109-43123. doi: 10.18632/oncotarget.10097.

37. Shi Z, Li X, Wu D, Tang R, Chen R, Xue S, Sun X. Silencing of HMGA2 suppresses cellular proliferation, migration, invasion, and epithelial-mesenchymal transition in bladder cancer. Tumour Biol. 2016; 37: 7515-23. doi: 10.1007/s13277-015-4625-2.

38. Iwaki $\mathrm{T}$, Urano $\mathrm{T}$, Umemura $\mathrm{K}$. PAI-1, progress in understanding the clinical problem and its aetiology. $\mathrm{Br} \mathrm{J}$ Haematol. 2012; 157: 291-8. doi: 10.1111/j.1365-2141.2012.09074.x.

39. Urquidi V, Kim J, Chang M, Dai Y, Rosser CJ, Goodison S. CCL18 in a multiplex urine-based assay for the detection of bladder cancer. PLoS One. 2012; 7: e37797. doi: 10.1371/ journal.pone.0037797.

40. Becker M, Szarvas T, Wittschier M, vom Dorp F, Totsch M, Schmid KW, Rubben H, Ergun S. Prognostic impact of plasminogen activator inhibitor type 1 expression in bladder cancer. Cancer. 2010; 116: 4502-12. doi: 10.1002/ cncr.25326.

41. Cordon-Cardo C, Sheinfeld J, Dalbagni G. Genetic studies and molecular markers of bladder cancer. Semin Surg Oncol. 1997; 13: 319-27.

42. George B, Datar RH, Wu L, Cai J, Patten N, Beil SJ, Groshen S, Stein J, Skinner D, Jones PA, Cote RJ. p53 gene and protein status: the role of p53 alterations in predicting outcome in patients with bladder cancer. J Clin Oncol. 2007; 25: 5352-8. doi: 10.1200/JCO.2006.10.4125.

43. Cordon-Cardo C, Zhang ZF, Dalbagni G, Drobnjak M, Charytonowicz E, Hu SX, Xu HJ, Reuter VE, Benedict WF. Cooperative effects of $\mathrm{p} 53$ and $\mathrm{pRB}$ alterations in primary superficial bladder tumors. Cancer Res. 1997; 57: 1217-21.

44. Shariat SF, Tokunaga H, Zhou J, Kim J, Ayala GE, Benedict WF, Lerner SP. p53, p21, pRB, and p16 expression predict clinical outcome in cystectomy with bladder cancer. J Clin Oncol. 2004; 22: 1014-24. doi: 10.1200/JCO.2004.03.118.

45. Stadler WM, Lerner SP, Groshen S, Stein JP, Shi SR, Raghavan D, Esrig D, Steinberg G, Wood D, Klotz L, Hall C, Skinner DG, Cote RJ. Phase III study of molecularly targeted adjuvant therapy in locally advanced urothelial cancer of the bladder based on p53 status. J Clin Oncol. 2011; 29: 3443-9. doi: 10.1200/JCO.2010.34.4028.

46. Sato H, Takino T. Coordinate action of membrane-type matrix metalloproteinase-1 (MT1-MMP) and MMP-2 enhances pericellular proteolysis and invasion. Cancer Sci. 2010; 101: 843-7. doi: 10.1111/j.1349-7006.2010.01498.x.

47. Schaal C, Pillai S, Chellappan SP. The Rb-E2F transcriptional regulatory pathway in tumor angiogenesis and metastasis. Adv Cancer Res. 2014; 121: 147-82. doi: 10.1016/B978-0-12-800249-0.00004-4.

48. Binder BR, Mihaly J, Prager GW. uPAR-uPA-PAI-1 interactions and signaling: a vascular biologist's view. Thromb Haemost. 2007; 97: 336-42.

49. Gabellini C, Del Bufalo D, Zupi G. Involvement of RB gene family in tumor angiogenesis. Oncogene. 2006; 25 : 5326-32. doi: 10.1038/sj.onc. 1209631.

50. Linden M, Segersten U, Runeson M, Wester K, Busch C, Pettersson U, Lind SB, Malmstrom PU. Tumour expression of bladder cancer-associated urinary proteins. BJU Int. 2013; 112: 407-15. doi: 10.1111/j.1464-410X.2012.11653.x.

51. Zhang G, Miyake M, Lawton A, Goodison S, Rosser CJ. Matrix metalloproteinase-10 promotes tumor progression through regulation of angiogenic and apoptotic pathways in cervical tumors. BMC Cancer. 2014; 14: 310. doi: 10.1186/1471-2407-14-310.

52. Miyake M, Lawton A, Goodison S, Urquidi V, GomesGiacoia E, Zhang G, Ross S, Kim J, Rosser CJ. Chemokine (C-X-C) ligand 1 (CXCL1) protein expression is increased in aggressive bladder cancers. BMC Cancer. 2013; 13: 322. doi: 10.1186/1471-2407-13-322. 